This item was submitted to Loughborough's Research Repository by the author.

Items in Figshare are protected by copyright, with all rights reserved, unless otherwise indicated.

\title{
Disturbance-observer-based robust control for time delay uncertain systems
}

PLEASE CITE THE PUBLISHED VERSION

http://link.springer.com/article/10.1007/s12555-010-0233-5?LI=true\#

\section{PUBLISHER}

(C) Institute of Control, Robotics and Systems Engineers (ICROS), Korean Institute of Electrical Engineers (KIEE) and Springer

\section{VERSION}

AM (Accepted Manuscript)

\section{LICENCE}

CC BY-NC-ND 4.0

\section{REPOSITORY RECORD}

Chen, Mou, and Wen-Hua Chen. 2012. "Disturbance-observer-based Robust Control for Time Delay Uncertain Systems". figshare. https://hdl.handle.net/2134/11161. 
This item was submitted to Loughborough's Institutional Repository (https://dspace.lboro.ac.uk/) by the author and is made available under the following Creative Commons Licence conditions.

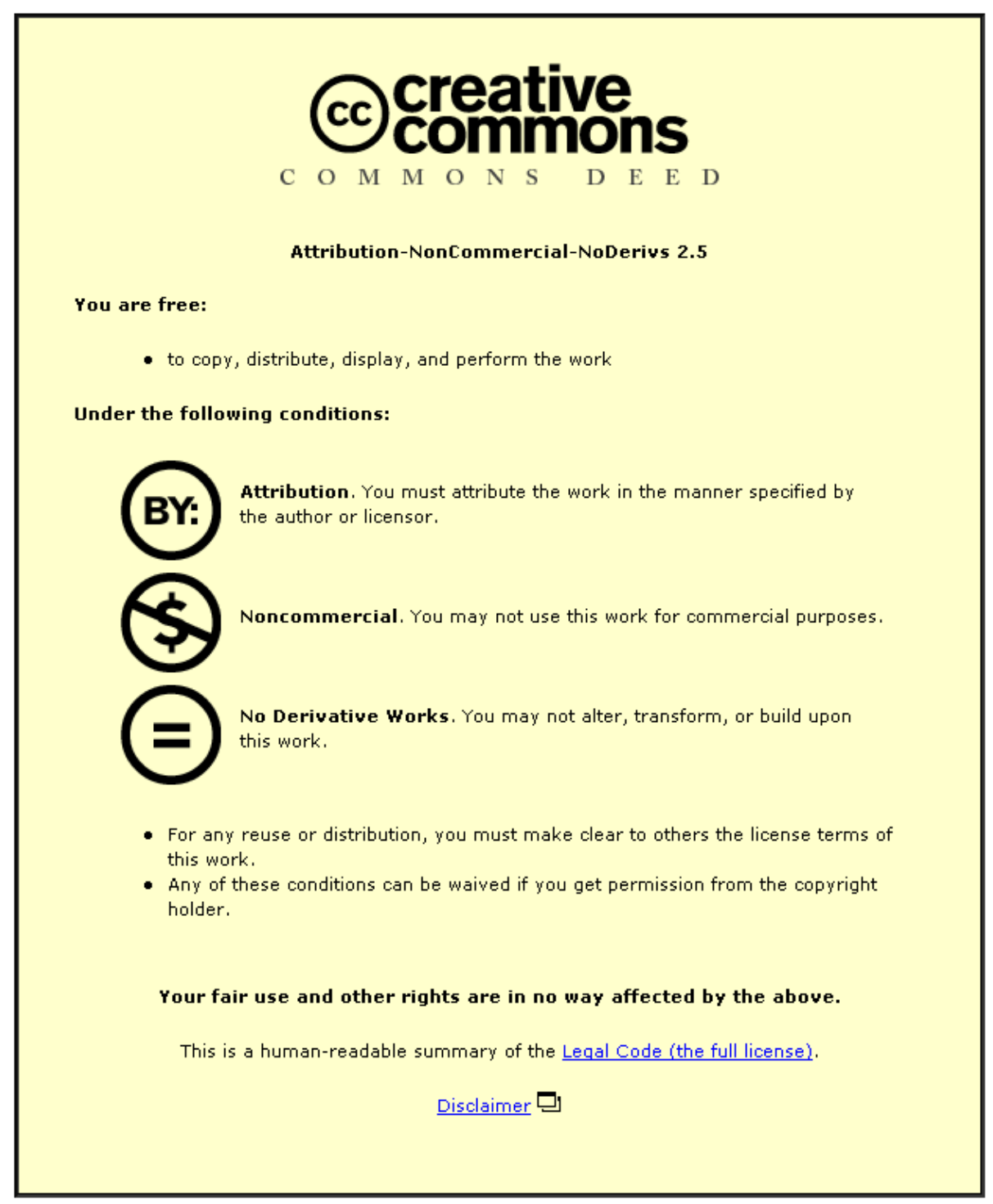

For the full text of this licence, please go to: http://creativecommons.org/licenses/by-nc-nd/2.5/ 


\title{
Disturbance-Observer-based Robust Control for Time Delay Uncertain Systems
}

\author{
Mou Chen ${ }^{1 *}$, Wen-Hua Chen ${ }^{2}$ \\ ${ }^{1}$ Automation College, Nanjing University of Aeronautics and Astronautics, Nanjing, 210016, China \\ 2 Department of Aeronautical and Automotive Engineering, Loughborough University, \\ Loughborough, Leicestershire LE11 3TU, UK
}

\begin{abstract}
A robust control scheme is proposed for a class of systems with uncertainty and time delay based on disturbance observer technique. A disturbance observer is developed to estimate the disturbance generated by an exogenous system, and the design parameter of the disturbance observer is determined by solving linear matrix inequalities (LMIs). Based on the output of the disturbance observer, a robust control scheme is proposed for the time delay uncertain system. The disturbance-observer-based robust controller combines two parts: one is a linear feedback controller designed using LMIs and the other is a compensatory controller designed with the output of the disturbance observer. By choosing an appropriate Lyapunov function candidate, the stability of the closed-loop system is proved. Finally, simulation example is presented to illustrate the effectiveness of the proposed control scheme.
\end{abstract}

Keywords: Uncertain system, Time delay, Disturbance observer, Robust control, Lyapunov method, LMI.

\section{Introduction}

As is well known, time delay often appears in many practical processes such as manual controls, neural networks, population dynamic models, rolling mills, and ship stabilization [1-8]. Furthermore, time-delay and uncertainties result in the instability and performance degradation of the closedloop system. Therefore, considerable attention has been paid to stability analysis and robust control design for time delay uncertain systems over the past years. Various effective techniques and their applications have been proposed, and their properties such as stability have been rigorously established.

${ }^{*}$ Corresponding author: chenmou@nuaa.edu.cn 
The guaranteed cost control was investigated for parameter uncertain systems with time delay in [1]. Kim [2] studied the robust stability of time-delayed linear systems with uncertainties. The problem of delay-dependent robust stability was investigated for systems with time-varying structured uncertainties and time-varying delays [3]. A robust controller was proposed in [4] for delay-dependent neutral systems with mixed delays and time-varying structured uncertainties. Sliding mode control scheme was presented for the robust stabilization of uncertain linear input-delay systems with nonlinear parametric perturbations in [5]. In [6], the stability of systems in the presence of bounded uncertain time-varying delays in the feedback loop was analysed. Han [7] studied the absolute stability for a class of nonlinear neutral systems using a discretized Lyapunov functional approach. In [8], an adaptive neural control scheme was proposed for a class of uncertain multi-input multi-output (MIMO) nonlinear state time-varying delay systems in a triangular control structure with unknown nonlinear dead-zones and gain signs.

In the controller design of time delay uncertain systems, among a few others, there are two most widely used methods: guaranteed cost control and $H_{\infty}$ control. The objective of guaranteed cost control is to design a feedback controller to stabilize a dynamic system and to provide an upper bound on the performance index in the presence of allowable uncertainty. The guaranteed cost control problem was studied for a class of linear time-delay systems via a memoryless state feedback control method in [9]. Lien [10] proposed a non-fragile guaranteed cost controller for a class of uncertain neutral system with time varying delays in both state and control input. The robust guaranteed cost controller was proposed for a class of uncertain neutral system with time-varying delays in [11]. In [12], a robust guaranteed cost control scheme for uncertain linear time-delay systems was proposed using dynamic output feedback.

In practical, except modeling error and parameter uncertainty, a time delay system may be subject to various external disturbances. The control of time delay uncertain systems with disturbance is an interesting topic. However, the guaranteed cost control is not sufficient to handle this kind control problem. Instead, the $H_{\infty}$ control scheme provide a very useful tool to address this problem. There have considerable research effort on $H_{\infty}$ control for uncertain time-delay systems. A $H_{\infty}$ control scheme for a class of linear systems with time-delays was proposed in [13]. Zhang and Han [14] studied the problem of delay-dependent robust $H_{\infty}$ filtering for uncertain linear systems with time-varying delay. In [15], a $H_{\infty}$ controller design method for continuous-time linear systems with time delay and actuator faults was investigated based on a LMI technique and an adaptive method. The issues of stability and $H_{\infty}$ control of linear systems with time-varying delays was considered in [16]. Kim and $\mathrm{Oh}$ [17] proposed the robust and non-fragile $H_{\infty}$ control for descriptor 
systems with parameter uncertainties and time delay. Delay-dependent robust $H_{\infty}$ control was proposed for uncertain systems with a state-delay in [18]. In [19], the robustness and $H_{\infty}$ control problems of output dynamic observer-based control for a class of uncertain linear systems with time delay was studied. The robust $H_{\infty}$ control scheme was proposed for linear time-delay systems with norm-bounded time-varying uncertainty in [20]. $H_{\infty}$ control have achieved widely regarded success. However the main drawbacks of this approach is that no information of the disturbance can be exploited within this framework. Further more, it is quite hard to directly address time domain specifications in tracking and regulation.

Many disturbances in real engineering are periodic and has inherit characteristic such as harmonics and unknown constant load. They can be modeled as output of a neutral stable exogenous system. This approach has been widely used in linear and nonlinear control such as internal model control, robust servo-regulator schemes and nonlinear regulation theory; for example [28], [21]. Over the last few years, considerable attention has been paid to the design of a disturbance observer to exploring the information about the characteristic of disturbances, where a disturbance observer can be used to approximate the system disturbance and a robust controller based on the output of the disturbance observer is designed to compensate the influence of unknown disturbances. Recently, using disturbance observers to study the robust control of nonlinear systems has been received increasing attention. Kim [22-23] proposed fuzzy disturbance observer and studied its application to control discrete-time and continuous-time systems. Chen [24] presented a general framework for nonlinear systems subject to disturbances using disturbance observer based control (DOBC) techniques. A new nonlinear PID predictive control scheme was proposed based on disturbance observers in [25]. These research results are applicable for systems whose disturbance relative degree is larger than or equal to their input relative degree, and disturbance is only present in one dimension. Applications have shown that disturbance observers can enhance disturbance attenuation and performance robustness. In [26], a nonlinear disturbance observer-based approach was proposed for longitudinal dynamics of a missile, while a new nonlinear disturbance observer for robotic manipulators was derived in [27]. Nevertheless, those research results did not consider a system with time delay.

This work is motivated by improving disturbance attenuation performance for robust control of time delay uncertain systems. To utilize the information of disturbances, a disturbance observer is developed to estimate disturbances generated by a linear exogenous system via linear matrix inequality (LMI). Using the output of the disturbance observer, a robust control scheme is developed. It is shown that zero steady state error can be achieved under the proposed scheme. The structure of the paper is as follows. The control problem for a class of time-delayed uncertain systems under 
disturbances is formulated in Section 2 and the design of disturbance observers is described in Section 3. Section 4 presents the design of a composite robust controller for a class of time-delayed uncertain systems using the output of disturbance observers, while the simulation results are given in Section 5 , followed by concluding remarks in Section 6.

\section{Problem description}

Consider a time delay uncertain system described in the form of

$$
\begin{aligned}
\dot{x}(t) & =[A+\Delta A(t)] x(t)+\left[A_{d}+\Delta A_{d}(t)\right] x(t-\tau(t))+B(u(t)+d(t)), t \geq 0 \\
x(t) & =\phi(t), t \in\left[-\tau_{M}, 0\right]
\end{aligned}
$$

where $x \in R^{n}$ is the state vector, $u \in R^{m}$ is the control input vector, and $d \in R^{m}$ is the system input disturbance with unknown boundary. $A, A_{d}$, and $B$ are constant matrices with corresponding dimensions. $\phi(t)$ is the initial vector of the system. The time delay, $\tau(t)$, is a time-varying continuous known function which satisfies

$$
0 \leq \tau(t) \leq \tau_{M}, \dot{\tau}(t) \leq \tau_{D}<1
$$

The uncertainties of system (1) are assumed to be of the form

$$
\left[\Delta A(t) \quad \Delta A_{d}(t)\right]=D F(t)\left[\begin{array}{ll}
E_{1} & E_{2}
\end{array}\right]
$$

where $D, E_{1}$ and $E_{2}$ are constant matrices with corresponding dimensions, representing the system structure uncertainty. $F(t)$ is an unknown, real and possibly time-varying matrix with Lebesguemeasurable elements satisfying

$$
F^{T}(t) F(t) \leq I, \forall t
$$

For the time delay uncertain system (1), a robust control can be designed using the $H_{\infty}$ control method. In $H_{\infty}$ setting, no information about the disturbances is required (except the noise has a limited power). Consequently, the disturbance attenuation results could be quite conservative. Similar to other work in robust servo regulator and nonlinear regulation theory, this paper consider a class of disturbance which can be modeled as output of an exogenous system. A disturbance observer is proposed to approximate the system disturbance, and a robust controller taking into account the output of the disturbance observer is designed for the time delay uncertain systems (1). The designed robust controller utilises estimate information of disturbance which results in a much 
improved disturbance attenuation ability. As will be shown in Section 6, in contrast to $H_{\infty}$ control, zero steady state error is achieved under the proposes scheme.

To proceed with the design of a robust controller for the time delay uncertain system, the following lemma is required.

Lemma 1: Assume that $X$ and $Y$ are vectors or matrices with appropriate dimension. The following inequality

$$
X^{T} Y+Y^{T} X \leq \alpha X^{T} X+\alpha^{-1} Y^{T} Y
$$

holds for any constant $\alpha>0$.

\section{Disturbance observer}

In this section, a disturbance observer is proposed for monitoring the disturbance of the uncertain time delay system (1). Suppose that the disturbance $d(t)$ of system (1) is generated by a linear exogenous system

$$
\left\{\begin{array}{l}
\dot{\xi}=W \xi \\
d=V \xi
\end{array}\right.
$$

where $\xi \in R^{n}$ and $d \in R^{m}$. W and $V$ are matrices with corresponding dimensions. As shown in [24], [28] and [21], a wide class of real engineering disturbance can be represented by this disturbance model (6). For example, for a unknown constant load disturbance, it can be represented by (6) with $W=0$ and $V=1$. For a harmonic disturbance with known frequency $\omega$ but unknown phase and magnitude, it can be represented by (6) with

$$
W=\left[\begin{array}{cc}
0 & \omega \\
-\omega & 0
\end{array}\right], \quad V=\left[\begin{array}{ll}
1 & 0
\end{array}\right]
$$

To estimate the unknown disturbance $d$ of the time delay system, a disturbance observer is proposed as follows.

$$
\left\{\begin{array}{l}
\dot{\varsigma}(t)=(W+L B V)(\varsigma(t)-L x(t))+L\left(A x(t)+A_{d} x(t-\tau(t))+B u\right) \\
\hat{\xi}(t)=\varsigma(t)-L x(t) \\
\hat{d}(t)=V \hat{\xi}(t)
\end{array}\right.
$$

where $L \in R^{n \times n}$ is a gain matrix which is a design parameter of disturbance observer which will be given by LMI. 
Define $e(t)=\xi(t)-\hat{\xi}(t)$. Differentiating $e(t)$ with respect to time, and considering (6) and (8) result in

$$
\dot{e}=\dot{\xi}(t)-\dot{\hat{\xi}}(t)=W \xi-(W+L B V)(\varsigma(t)-L x(t))-L\left(A x(t)+A_{d} x(t-\tau(t))+B u\right)+L \dot{x}
$$

Considering (6) and (8), and invoking (1), we obtain

$$
\begin{aligned}
\dot{e} & =W \xi-(W+L B V)(\varsigma(t)-L x(t))-L\left(A x(t)+A_{d} x(t-\tau(t))+B u\right) \\
& +L[A+\Delta A(t)] x(t)+L\left[A_{d}+\Delta A_{d}(t)\right] x(t-\tau(t))+L B u+L B d(t) \\
& =(W+L B V) e(t)+L \Delta A(t) x(t)+L \Delta A_{d} x(t-\tau(t))
\end{aligned}
$$

The objective of disturbance approximation can be achieved by designing the observer gain matrix $L$ such that Eq.(10) satisfies the desired stability and robustness performance.

Remark 1: It can be seem form Eq.(10) that $L$ is an important design parameter of the disturbance observer (8). The choice of $L$ has influence not only on the stability of the observer, i.e. $W+L B V<0$, but also on robust performance under the uncertainties $L \Delta A(t) x(t)$ and $L \Delta A_{d}(t) x(t-\tau(t))$.

\section{Design of disturbance-observer-based robust controller}

In this section, the robust controller is proposed based on the disturbance observer. Suppose that the system states can be directly measured. Then the robust controller can be designed as

$$
u(t)=u_{k}(t)+u_{c}(t)
$$

where

$$
\begin{gathered}
u_{k}(t)=K x(t) \\
u_{c}(t)=-\hat{d}
\end{gathered}
$$

where $u_{k}$ is the linear state feedback controller, and $u_{c}$ is a compensatory controller. Matrix $K$ will be given by solving the LMI. $\hat{d}$ is the approximation of the system disturbance $d$, and it is the output of disturbance observer (8).

The main task is now to design disturbance gain matrix $L$, and feedback gain matrix $K$ such that the closed-loop system states and disturbance observer approximation error are asymptotically stable. For the disturbance observer and robust controller proposed in (8) and (11) respectively, the stability condition is given in the following theorem. 
Theorem 1: For given positive constants $\alpha_{i}, i=1,2,3,4$, if there exist some matrices $X \in$ $R^{n \times n}>0, \bar{P}_{1} \in R^{n \times n}>0, P_{2} \in R^{n \times n}>0, H \in R^{n \times n}, Y \in R^{m \times n}, \bar{Q}_{1} \in R^{n \times n}, \bar{Q}_{2} \in R^{n \times n}$, $\bar{Q}_{3} \in R^{n \times n}$ and $\bar{Q}_{4} \in R^{n \times n}$ such that the following LMI hold:

$$
\left[\begin{array}{ccccccc}
\Lambda_{11} & \Lambda_{12} & \Lambda_{13} & \Lambda_{14} & X E_{1}^{T} & 0 & 0 \\
\Lambda_{12}^{T} & \Lambda_{22} & \Lambda_{23} & \Lambda_{24} & 0 & H D & 0 \\
\Lambda_{13}^{T} & \Lambda_{23}^{T} & \Lambda_{33} & \Lambda_{34} & 0 & 0 & X E_{2}^{T} \\
\Lambda_{14}^{T} & \Lambda_{24}^{T} & \Lambda_{34}^{T} & \Lambda_{44} & 0 & 0 & 0 \\
E_{1} X & 0 & 0 & 0 & -\left(\alpha_{1}^{-1}+\alpha_{3}^{-1}\right)^{-1} I & 0 & 0 \\
0 & D^{T} H^{T} & 0 & 0 & 0 & -\left(\alpha_{3}+\alpha_{4}\right)^{-1} I & 0 \\
0 & 0 & E_{2} X & 0 & 0 & 0 & -\left(\alpha_{2}^{-1}+\alpha_{4}^{-1}\right)^{-1} I
\end{array}\right]<0
$$

where $\Lambda_{11}=A X+B Y+X A^{T}+Y^{T} B^{T}+\bar{P}_{1}-\bar{Q}_{1}-\bar{Q}_{1}^{T}+\left(\alpha_{1}+\alpha_{2}\right) D D^{T}, \Lambda_{12}=B V-\bar{Q}_{4}$, $\Lambda_{13}=A_{d} X-\bar{Q}_{2}+\bar{Q}_{1}^{T}, \Lambda_{14}=-\bar{Q}_{3}+\bar{Q}_{1}^{T}, \Lambda_{22}=P_{2} W+W^{T} P_{2}+H B V+V^{T} B^{T} H^{T}, \Lambda_{23}=\bar{Q}_{4}^{T}$, $\Lambda_{24}=\bar{Q}_{4}^{T}, \Lambda_{33}=\left(\tau_{D}-1\right) \bar{P}_{1}+\bar{Q}_{2}+\bar{Q}_{2}^{T}, \Lambda_{34}=\bar{Q}_{3}+\bar{Q}_{2}^{T}, \Lambda_{44}=\bar{Q}_{3}+\bar{Q}_{3}^{T}$. Then the closed-loop system states and disturbance observer approximation error are asymptotically stable under the proposed composite control law (11) when selecting $K=Y X^{-1}$ and $L=P_{2}^{-1} H$.

Proof: Let the Lyapunov function candidate be given by

$$
V(x(t), e(t))=x^{T}(t) P x(t)+\int_{t-\tau(t)}^{t} x^{T}(s) P_{1} x(s) d s+e^{T}(t) P_{2} e(t)
$$

where $P=X^{-1}$ and $P_{1}=X^{-1} \bar{P}_{1} X^{-1}$ are positive-definite matrices with corresponding dimensions.

Substituting (11), (12) and (13) into (1) yield

$$
\begin{array}{r}
\dot{x}(t)=(A+\Delta A) x(t)+\left(A_{d}+\Delta A_{d}\right) x(t-\tau(t))+B\left(K x(t)+u_{c}\right)+B d(t) \\
=(A+\Delta A+B K) x(t)+\left(A_{d}+\Delta A_{d}\right) x(t-\tau(t))-B \hat{d}+B d(t)
\end{array}
$$

According to Leibniz-Newton formula

$$
\int_{t-\tau}^{t} \dot{x}(s) d s-x(t)+x(t-\tau)=0
$$

the time derivatives of $V(x(t), e(t))$, along the trajectories of system (1), is given by

$$
\begin{aligned}
\dot{V} & =x^{T}(t)\left[P(A+\Delta A+B K)+(A+\Delta A+B K)^{T} P\right] x(t)+2 x^{T}(t) P\left(A_{d}+\Delta A_{d}\right) x(t-\tau) \\
& +2 x^{T}(t) P B d(t)-2 x^{T}(t) P B \hat{d}(t)+x^{T}(t) P_{1} x(t)-(1-\dot{\tau}) x^{T}(t-\tau) P_{1} x(t-\tau)+2 e^{T} P_{2} \dot{e} \\
& +\left[\int_{t-\tau}^{t} \dot{x}(s) d s-x(t)+x(t-\tau)\right]^{T}\left[Q_{1} x(t)+Q_{2} x(t-\tau)+Q_{3} \int_{t-\tau}^{t} \dot{x}(s) d s+Q_{4} e(t)\right] \\
& +\left[Q_{1} x(t)+Q_{2} x(t-\tau)+Q_{3} \int_{t-\tau}^{t} \dot{x}(s) d s+Q_{4} e(t)\right]^{T}\left[\int_{t-\tau}^{t} \dot{x}(s) d s-x(t)+x(t-\tau)\right]
\end{aligned}
$$


Define $\tilde{d}=d-\hat{d}$. Substituting (10) into (18) yields

$$
\begin{aligned}
\dot{V} & =x^{T}(t)\left[P(A+B K)+(A+B K)^{T} P\right] x(t)+x^{T}(t)\left[P \Delta A+\Delta A^{T} P\right] x(t)+x^{T}(t) P A_{d} x(t-\tau) \\
& +x^{T}(t-\tau) A_{d}^{T} P x(t)+x^{T} P \Delta A_{d} x(t-\tau)+x^{T}(t-\tau) \Delta A_{d}^{T} P x(t)+x^{T}(t) P B \tilde{d}(t) \\
& +\tilde{d}^{T}(t) B^{T} P x(t)+x^{T}(t) P_{1} x(t)-(1-\dot{\tau}) x^{T}(t-\tau) P_{1} x(t-\tau) \\
& +\left[\int_{t-\tau}^{t} \dot{x}(s) d s-x(t)+x(t-\tau)\right]^{T}\left[Q_{1} x(t)+Q_{2} x(t-\tau)+Q_{3} \int_{t-\tau}^{t} \dot{x}(s) d s+Q_{4} e(t)\right] \\
& +\left[Q_{1} x(t)+Q_{2} x(t-\tau)+Q_{3} \int_{t-\tau}^{t} \dot{x}(s) d s+Q_{4} e(t)\right]^{T}\left[\int_{t-\tau}^{t} \dot{x}(s) d s-x(t)+x(t-\tau)\right] \\
& +e^{T}(t)\left[P_{2}(W+L B V)+(W+L B V)^{T} P_{2}\right] e(t)+e^{T}(t) P_{2} L \Delta A x(t)+x^{T}(t) \Delta A^{T} L^{T} P_{2} e \\
& +e^{T}(t) P_{2} L \Delta A_{d} x(t-\tau)+x^{T}(t-\tau) \Delta A_{d}^{T} L^{T} P \in e(t)
\end{aligned}
$$

Recalling (6) and (8), it obtains

$$
\tilde{d}=d-\hat{d}=V \xi(t)-V \hat{\xi}(t)=V e
$$

Considering (3) and substituting (20) into (19), we have

$$
\begin{aligned}
\dot{V} & =x^{T}(t)\left[P(A+B K)+(A+B K)^{T} P\right] x(t)+x^{T}(t)\left[P D F E_{1}+E_{1}^{T} F^{T} D^{T} P\right] x(t) \\
& +x^{T}(t) P A_{d} x(t-\tau)+x^{T}(t)(t-\tau) A_{d}^{T} P x(t)+x^{T}(t) P D F E_{2} x(t-\tau) \\
& +x^{T}(t-\tau) E_{2}^{T} F^{T} D^{T} P x(t)+x^{T}(t) P B V e(t)+e^{T}(t) V^{T} B^{T} P x(t) \\
& +x^{T}(t) P_{1} x(t)-(1-\dot{\tau}) x^{T}(t-\tau) P_{1} x(t-\tau) \\
& +\left[\int_{t-\tau}^{t} \dot{x}(s) d s-x(t)+x(t-\tau)\right]^{T}\left[Q_{1} x(t)+Q_{2} x(t-\tau)+Q_{3} \int_{t-\tau}^{t} x(s) d s+Q_{4} e(t)\right] \\
& +\left[Q_{1} x(t)+Q_{2} x(t-\tau)+Q_{3} \int_{t-\tau}^{t} \dot{x}(s) d s+Q_{4} e(t)\right]^{T}\left[\int_{t-\tau}^{t} \dot{x}(s) d s-x(t)+x(t-\tau)\right] \\
& +e^{T}(t)\left[P_{2}(W+L B V)+(W+L B V)^{T} P_{2}\right] e(t)+e^{T}(t) P_{2} L D F E_{1} x(t) \\
& +x^{T}(t) E_{1}^{T} F^{T} D^{T} L^{T} P_{2} e(t)+e^{T}(t) P_{2} L D F E_{2} x(t-\tau)+x^{T}(t-\tau) E_{2}^{T} F^{T} D^{T} L^{T} P_{2} e(t)
\end{aligned}
$$

Using Lemma 1, one can show that

$$
\begin{aligned}
& x^{T}(t)\left[P D F E_{1}+E_{1}^{T} F^{T} D^{T} P\right] x(t) \leq \alpha_{1} x^{T}(t) P D D^{T} P x(t)+\alpha_{1}^{-1} x^{T}(t) E_{1}^{T} E_{1} x(t) \\
& x^{T}(t) P D F E_{2} x(t-\tau)+x^{T}(t-\tau) E_{2}^{T} F^{T} D^{T} P x(t) \\
& \leq \alpha_{2} x^{T}(t) P D D^{T} P x(t)+\alpha_{2}^{-1} x^{T}(t-\tau) E_{2}^{T} E_{2} x(t-\tau) \\
& e^{T}(t) P_{2} L D F E_{1} x(t)+x^{T}(t) E_{1}^{T} F^{T} D^{T} L^{T} P_{2} e(t) \\
& \leq \alpha_{3} e^{T}(t) P_{2} L D D^{T} L^{T} P_{2} e(t)+\alpha_{3}^{-1} x^{T}(t) E_{1}^{T} E_{1} x(t)
\end{aligned}
$$




$$
\begin{aligned}
e^{T}(t) P_{2} L D F E_{2} x(t-\tau) & +x^{T}(t-\tau) E_{2}^{T} F^{T} D^{T} L^{T} P_{2} e(t) \\
& \leq \alpha_{4} e^{T}(t) P_{2} L D D^{T} L^{T} P_{2} e(t)+\alpha_{4}^{-1} x^{T}(t-\tau) E_{2}^{T} E_{2} x(t-\tau)
\end{aligned}
$$

Substituting (22)-(25) into (21) gives

$$
\begin{aligned}
\dot{V} & \leq x^{T}(t)\left[P(A+B K)+(A+B K)^{T} P\right] x(t)+\alpha_{1} x^{T}(t) P D D^{T} P x(t) \\
& +\alpha_{1}^{-1} x^{T}(t) E_{1}^{T} E_{1} x(t)+x^{T}(t) P A_{d} x(t-\tau)+x^{T}(t-\tau) A_{d}^{T} P x(t) \\
& +\alpha_{2} x^{T}(t) P D D^{T} P x(t)+\alpha_{2}^{-1} x^{T}(t-\tau) E_{2}^{T} E_{2} x(t-\tau)+x^{T}(t) P B V e(t) \\
& +e^{T}(t) V^{T} B^{T} P x(t)+x^{T}(t) P_{1} x(t)-(1-\dot{\tau}) x^{T}(t-\tau) P_{1} x(t-\tau) \\
& +\left[\int_{t-\tau}^{t} \dot{x}(s)-x(t)+x(t-\tau)\right]^{T}\left[Q_{1} x(t)+Q_{2} x(t-\tau)+Q_{3} \int_{t-\tau}^{t} x(s) d s+Q_{4} e(t)\right] \\
& +\left[Q_{1} x(t)+Q_{2} x(t-\tau)+Q_{3} \int_{t-\tau}^{t} \dot{x}(s) d s+Q_{4} e(t)\right]^{T}\left[\int_{t-\tau}^{t} \dot{x}(s)-x(t)+x(t-\tau)\right] \\
& +e^{T}(t)\left[P_{2}(W+L B V)+(W+L B V)^{T} P_{2}\right] e(t)+\alpha_{3} e^{T}(t) P_{2} L D D^{T} L^{T} P_{2} e(t) \\
& +\alpha_{3}^{-1} x^{T}(t) E_{1}^{T} E_{1} x(t)+\alpha_{4} e^{T}(t) P_{2} L D D^{T} L^{T} P_{2} e(t)+\alpha_{4}^{-1} x^{T}(t-\tau) E_{2}^{T} E_{2} x(t-\tau)
\end{aligned}
$$

Eq.(26) can be rewritten as

$$
\dot{V} \leq\left[\begin{array}{c}
x(t) \\
e(t) \\
x(t-\tau) \\
\int_{t-\tau}^{t} \dot{x}(s) d s
\end{array}\right]^{T} \bar{\Omega}_{0}\left[\begin{array}{c}
x(t) \\
e(t) \\
x(t-\tau) \\
\int_{t-\tau}^{t} \dot{x}(s) d s
\end{array}\right]
$$

where

$$
\bar{\Omega}_{0}=\left[\begin{array}{cccc}
\bar{\Lambda}_{11} & \bar{\Lambda}_{12} & \bar{\Lambda}_{13} & \bar{\Lambda}_{14} \\
\bar{\Lambda}_{12}^{T} & \bar{\Lambda}_{22} & \bar{\Lambda}_{23} & \bar{\Lambda}_{24} \\
\bar{\Lambda}_{13}^{T} & \bar{\Lambda}_{23}^{T} & \bar{\Lambda}_{33} & \bar{\Lambda}_{34} \\
\bar{\Lambda}_{14}^{T} & \bar{\Lambda}_{24}^{T} & \bar{\Lambda}_{34}^{T} & \bar{\Lambda}_{44}
\end{array}\right]
$$

where $\bar{\Lambda}_{11}=P(A+B K)+(A+B K)^{T} P+P_{1}-Q_{1}-Q_{1}^{T}+\left(\alpha_{1}+\alpha_{2}\right) P D D^{T} P+\alpha_{1}^{-1} E_{1}^{T} E_{1}+\alpha_{3}^{-1} E_{1}^{T} E_{1}$, $\bar{\Lambda}_{12}=P B V-Q_{4}, \bar{\Lambda}_{13}=P A_{d}-Q_{2}+Q_{1}^{T}, \bar{\Lambda}_{14}=-Q_{3}+Q_{1}^{T}, \bar{\Lambda}_{22}=P_{2}(W+L B V)+(W+L B V)^{T} P_{2}+$ $\left(\alpha_{3}+\alpha_{4}\right) P_{2} L D D^{T} L^{T} P_{2}, \bar{\Lambda}_{23}=Q_{4}^{T} \bar{\Lambda}_{24}=Q_{4}^{T}, \bar{\Lambda}_{33}=\left(\tau_{D}-1\right) P_{1}+Q_{2}+Q_{2}^{T}+\alpha_{2}^{-1} E_{2}^{T} E_{2}+\alpha_{4}^{-1} E_{2}^{T} E_{2}$, $\bar{\Lambda}_{34}=Q_{3}+Q_{2}^{T}, \bar{\Lambda}_{44}=Q_{3}+Q_{3}^{T}$.

Suppose that

$$
\begin{aligned}
P & =X^{-1}, K=Y X^{-1}, P_{1}=P \bar{P}_{1} P, L=P_{2}^{-1} H, Q_{1}=P \bar{Q}_{1} P \\
Q_{2} & =P \bar{Q}_{2} P, Q_{3}=P \bar{Q}_{3} P, Q_{4}=P \bar{Q}_{4}
\end{aligned}
$$


Left and right multiplying both sides of $(28)$ by $\operatorname{diag}\left\{P^{-1}, I, P^{-1}, P^{-1}\right\}$, one obtains

$$
\Omega_{0}=\left[\begin{array}{cccc}
\overline{\bar{\Lambda}}_{11} & \overline{\bar{\Lambda}}_{12} & \overline{\bar{\Lambda}}_{13} & \overline{\bar{\Lambda}}_{14} \\
\overline{\bar{\Lambda}}_{12}^{T} & \overline{\bar{\Lambda}}_{22} & \overline{\bar{\Lambda}}_{23} & \overline{\bar{\Lambda}}_{24} \\
\overline{\bar{\Lambda}}_{13}^{T} & \overline{\bar{\Lambda}}_{23}^{T} & \overline{\bar{\Lambda}}_{33} & \overline{\bar{\Lambda}}_{34} \\
\overline{\bar{\Lambda}}_{14}^{T} & \overline{\bar{\Lambda}}_{24}^{T} & \overline{\bar{\Lambda}}_{34}^{T} & \overline{\bar{\Lambda}}_{44}
\end{array}\right]
$$

where $\overline{\bar{\Lambda}}_{11}=A X+B Y+X A^{T}+Y^{T} B^{T}+\bar{P}_{1}-\bar{Q}_{1}-\bar{Q}_{1}^{T}+\left(\alpha_{1}+\alpha_{2}\right) D D^{T}+\left(\alpha_{1}^{-1}+\alpha_{3}^{-1}\right) X E_{1}^{T} E_{1} X$, $\overline{\bar{\Lambda}}_{12}=B V-\bar{Q}_{4}, \overline{\bar{\Lambda}}_{13}=A_{d} X-\bar{Q}_{2}+\bar{Q}_{1}^{T}, \overline{\bar{\Lambda}}_{14}=-\bar{Q}_{3}+\bar{Q}_{1}^{T}, \overline{\bar{\Lambda}}_{22}=P_{2} W+W^{T} P_{2}+H B V+V^{T} B^{T} H^{T}+$ $\left(\alpha_{3}+\alpha_{4}\right) H D D^{T} H^{T}, \overline{\bar{\Lambda}}_{23}=\bar{Q}_{4}^{T} \overline{\bar{\Lambda}}_{24}=\bar{Q}_{4}^{T}, \overline{\bar{\Lambda}}_{33}=\left(\tau_{D}-1\right) \bar{P}_{1}+\bar{Q}_{2}+\bar{Q}_{2}^{T}+\left(\alpha_{2}^{-1}+\alpha_{4}^{-1}\right) X E_{2}^{T} E_{2} X$, $\overline{\bar{\Lambda}}_{34}=\bar{Q}_{3}+\bar{Q}_{2}^{T}, \overline{\bar{\Lambda}}_{44}=\bar{Q}_{3}+\bar{Q}_{3}^{T}$.

Eq.(30) can be rewritten as

$$
\Omega_{0}=\left[\begin{array}{cccc}
\Lambda_{11} & \Lambda_{12} & \Lambda_{13} & \Lambda_{14} \\
\Lambda_{12}^{T} & \Lambda_{22} & \Lambda_{23} & \Lambda_{24} \\
\Lambda_{13}^{T} & \Lambda_{23}^{T} & \Lambda_{33} & \Lambda_{34} \\
\Lambda_{14}^{T} & \Lambda_{24}^{T} & \Lambda_{34}^{T} & \Lambda_{44}
\end{array}\right]+\Delta
$$

where

$\Delta=\left[\begin{array}{ccc}X E_{1}^{T} & 0 & 0 \\ 0 & H D & 0 \\ 0 & 0 & X E_{2}^{T}\end{array}\right]\left[\begin{array}{ccc}\left(\alpha_{1}^{-1}+\alpha_{3}^{-1}\right) I & 0 & 0 \\ 0 & \left(\alpha_{3}+\alpha_{4}\right) I & 0 \\ 0 & 0 & \left(\alpha_{2}^{-1}+\alpha_{4}^{-1}\right)\end{array}\right]\left[\begin{array}{ccc}E_{1} X & 0 & 0 \\ 0 & D^{T} H^{T} & 0 \\ 0 & 0 & E_{2} X\end{array}\right]$.

Considering (14) and applying the Schur complement theorem, it can be shown that

$$
\Omega_{0}<0
$$

Combining (27), (28), (30), (31) with (32) reaches

$$
\dot{V}<0
$$

It follows from Eq.(33) that the closed loop system states of the time delay uncertain system and disturbance observer approximation error are asymptotically stable. Hence, the convergence of $x$ and $e$ is proven using the Lyapunov stability criterion.

Remark 2: Solving the inequality (14) yields the feedback gain matrix $K$ and disturbance observer gain matrix L. Eq.(14) is a LMI after $\alpha_{i}, i=1,2,3,4$ are chosen. This will introduce conservativeness with a given set of $\alpha_{i}$. The conservativeness can be reduced by using linear search techniques. The feedback gain matrix $K$ and disturbance observer matrix $L$ satisfying Eq.(14) 
guarantee the convergence of the disturbance observer estimate error and zero state state error of the system states.

Remark 3: A delay-independent stabilization control is developed in this paper. All the existing robust control methods and stability conditions for time delay systems can be classified into delayindependent and delay-dependent categories. As it is well known, delay-independent methods give results irrespective of the size of delay, while the delay-dependent results for time delay systems are dependent of the size of delay. In general, the delay-dependent stabilization is considered to be less conservative than the delay-independent one.

\section{Simulation example}

For illustrating the effectiveness of the proposed control scheme, an example is given to show the effectiveness in this section.

The longitudinal dynamics of a fighter at $H=2800 \mathrm{ft}$ and $V=0.5 \mathrm{M}$ can be expressed as

$$
\left[\begin{array}{c}
\dot{\theta} \\
\ddot{\theta} \\
\dot{\alpha}
\end{array}\right]=(A+\Delta A(t))\left[\begin{array}{c}
\theta \\
\dot{\theta} \\
\alpha
\end{array}\right]+\left[A_{d}+\Delta A_{d}(t)\right] x(t-\tau(t))+B\left(\left[\begin{array}{c}
\delta_{z 1} \\
\delta_{z 2}
\end{array}\right]+d(t)\right)
$$

where $x=[\theta, \dot{\theta}, \alpha]^{T}, \theta, \dot{\theta}$ and $\alpha$ denote pitching angle, pitching rate and angle of attack, respectively. $\delta_{z 1}$ and $\delta_{z 2}$ stand elevator and flap deflection angle, respectively. When time delay and uncertainty are considered, Eq.(34) can be written as the form of system (1), where

$$
\begin{aligned}
& A=\left[\begin{array}{ccc}
1 & 0 & 0 \\
1 & -0.87 & 43.2 \\
0 & 0.99 & -1.34
\end{array}\right], A_{d}=\left[\begin{array}{ccc}
0.8 & 0 & 0 \\
0 & -0.87 & 0 \\
0 & 0.99 & -1
\end{array}\right], B=\left[\begin{array}{cc}
1 & 0 \\
-17.25 & -1.58 \\
-0.17 & -0.25
\end{array}\right] \\
& D=\left[\begin{array}{ccc}
1 & 0 & 0 \\
0 & 1 & 0 \\
0 & 0 & 1
\end{array}\right], F=\left[\begin{array}{ccc}
\sin (t) / 2 & 0 & 0 \\
0 & \cos (t) / 2 & 0 \\
0 & 0 & \sin (t) / 2
\end{array}\right] \\
& E_{1}=\left[\begin{array}{ccc}
0.2 & 0 & 0 \\
0 & 0.2 & 0
\end{array}\right], E_{2}=\left[\begin{array}{ccc}
0.3 & 0 & 0 \\
0 & 0.3 & 0 \\
0 & 0 & 0.3
\end{array}\right], \tau_{M}=1, \tau_{D}=0.5
\end{aligned}
$$


The system disturbance $d(t)$ can be generated by a linear exogenous neutral stable system described by (6) with

$$
W=\left[\begin{array}{ccc}
0 & 1.5 & 0 \\
-1.5 & 0 & 0 \\
0 & 0 & -1.5
\end{array}\right], V=\left[\begin{array}{lll}
1 & 0 & 0 \\
0 & 1 & 0
\end{array}\right]
$$

which represents an external periodic disturbance with known frequency but without any information of its magnitude and phase.

With $\alpha_{1}=\alpha_{2}=\alpha_{3}=\alpha_{4}=1$, solving LMI (14) gives

$$
\begin{aligned}
& X=\left[\begin{array}{ccc}
0.9948 & 0.0053 & -0.8577 \\
0.0053 & 1.4813 & 0.2789 \\
-0.8577 & 0.2789 & 3.1306
\end{array}\right], \bar{P}_{1}=\left[\begin{array}{ccc}
4.5197 & -0.2276 & 0.9174 \\
-0.2276 & 8.3131 & 0.1340 \\
0.9174 & 0.1340 & 8.5229
\end{array}\right], \\
& P_{2}=\left[\begin{array}{ccc}
5.1318 & 1.5042 & 0.0211 \\
1.5042 & 5.7386 & 0.0549 \\
0.0211 & 0.0549 & 2.5446
\end{array}\right], H=\left[\begin{array}{ccc}
-0.0621 & 0.6382 & 0.0061 \\
0.6382 & 0.1058 & -0.0014 \\
0.0061 & -0.0014 & 0.0006
\end{array}\right] \\
& \bar{Q}_{1}=\left[\begin{array}{ccc}
-0.0236 & 0.0841 & -0.6509 \\
0.0841 & -2.1655 & -0.2406 \\
-0.6509 & -0.2406 & -1.0964
\end{array}\right], \bar{Q}_{2}=\left[\begin{array}{ccc}
-0.3248 & 0.0271 & 0.2028 \\
0.0271 & -0.4675 & -0.0972 \\
0.2028 & -0.0972 & -1.3232
\end{array}\right], \\
& \bar{Q}_{3}=\left[\begin{array}{ccc}
-1.3548 & 0.0276 & -0.1409 \\
0.0276 & -1.3193 & -0.0166 \\
-0.1409 & -0.0166 & -1.2556
\end{array}\right], \bar{Q}_{4}=\left[\begin{array}{ccc}
0.1916 & -1.1576 & -0.1207 \\
-1.1576 & -0.2321 & -0.0523 \\
-0.1207 & -0.0523 & -0.0028
\end{array}\right], \\
& Y=\left[\begin{array}{ccc}
-6.0529 & -8.2356 & 4.1912 \\
36.9789 & 107.8751 & 23.7502
\end{array}\right], K=\left[\begin{array}{ccc}
-5.8547 & -5.5824 & 0.2322 \\
49.7211 & 69.8247 & 14.9872
\end{array}\right], \\
& L=\left[\begin{array}{ccc}
-0.0484 & 0.1289 & 0.0014 \\
0.1239 & -0.0153 & -0.0006 \\
0.0001 & -0.0013 & 0.0003
\end{array}\right], W+L B V=\left[\begin{array}{ccc}
-2.2714 & 1.2961 & 0 \\
-1.1115 & 0.0244 & 0 \\
0.0223 & 0.0020 & -1.5000
\end{array}\right]
\end{aligned}
$$

The initial state values are $x_{0}=[0.1,-0.1,0]^{T}$, the initial generated disturbance value are $d_{0}=[-0.12,-0.13]^{T}$, and the disturbance observer initial value are $\hat{d}_{0}=[-0.1,-0.1]^{T}$. The robust 


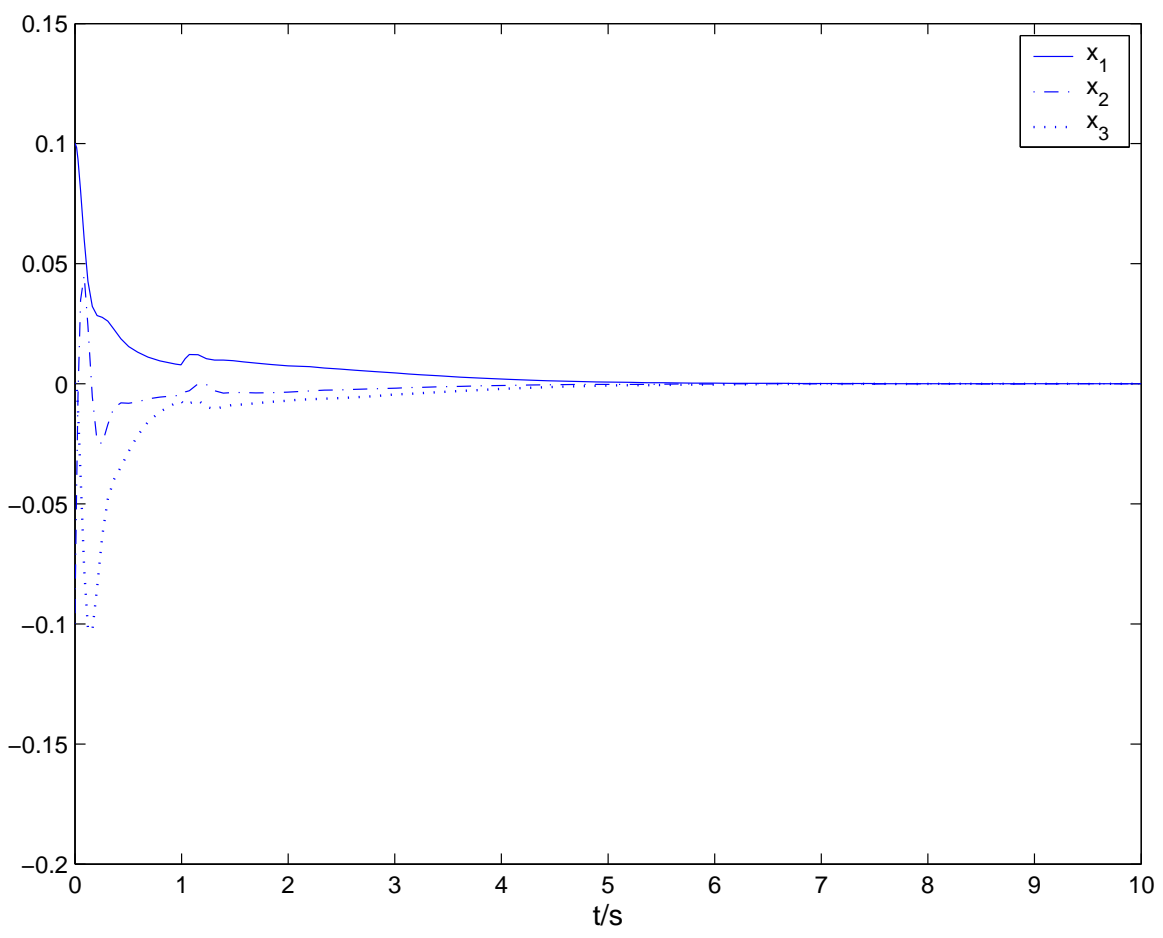

Figure 1: The closed-loop state response

controller is designed according to (11), and the simulation results are shown in Fig.1-Fig.3. It is shown in Fig. 1 that the stability of the closed-loop system can be obtained under the proposed robust controller. The simulation results shown in Figs. 2 and 3 indicate that the output of disturbance can effectively approximate the unknown external harmonic disturbance.

From these simulation results of the example, we can know that the disturbance observer can well approximate the system disturbance, and the designed robust control scheme based on disturbance observer is valid.

\section{Conclusion}

In this paper, a disturbance-observer-based robust controller is proposed for a class of time delay uncertain systems. To enhance the disturbance attenuation and performance robustness, the disturbance observer is designed, and it can be used to approximate the system disturbance which is generated by a linear exogenous system. Based on the output of the disturbance observer, a robust controller is presented for the time delay uncertain system, and the stability is proved of the closedloop system using Lyapunov method. Finally, an example is used to illustrate the effectiveness of the proposed robust control scheme. The simulation result suggests that the designed robust control 


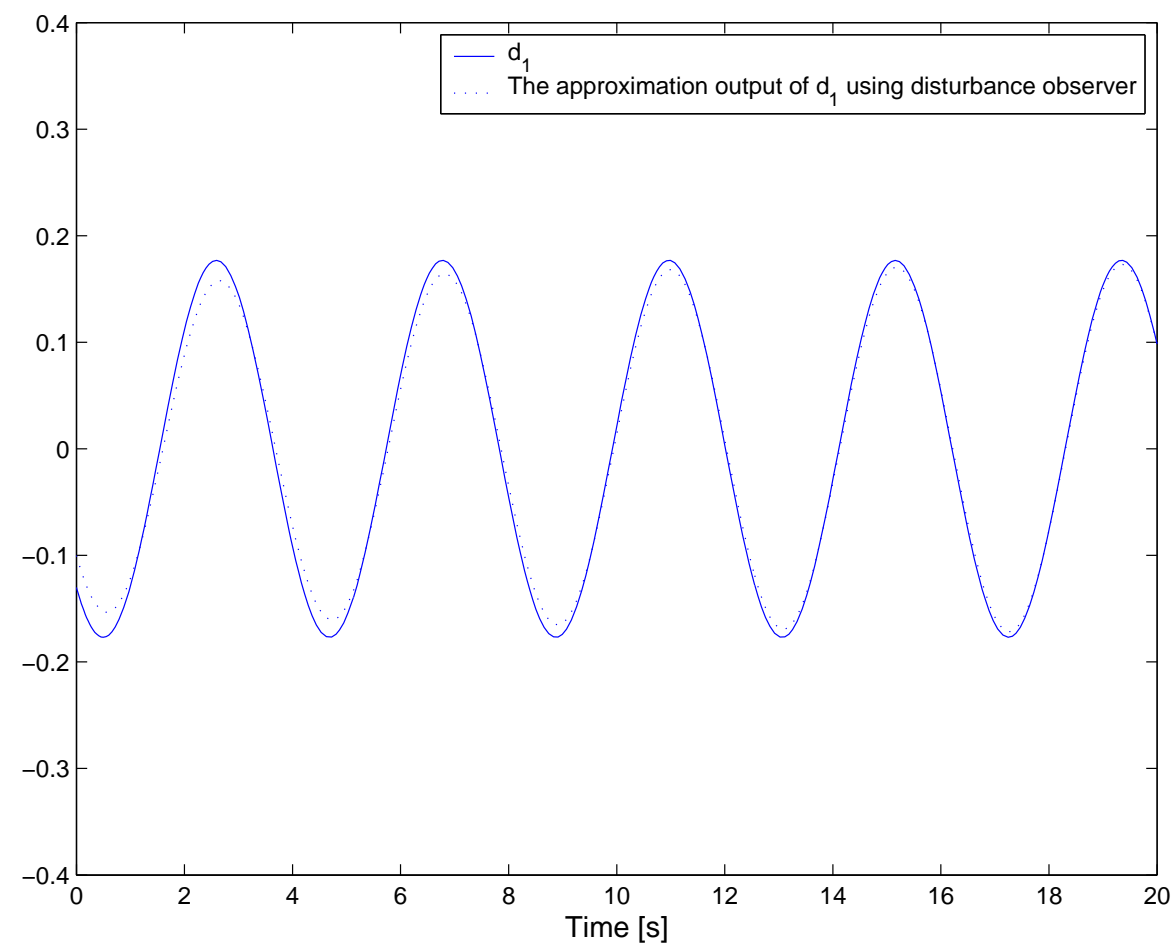

Figure 2: The disturbance $d_{1}$ and the approximation output of $\hat{d}_{1}$

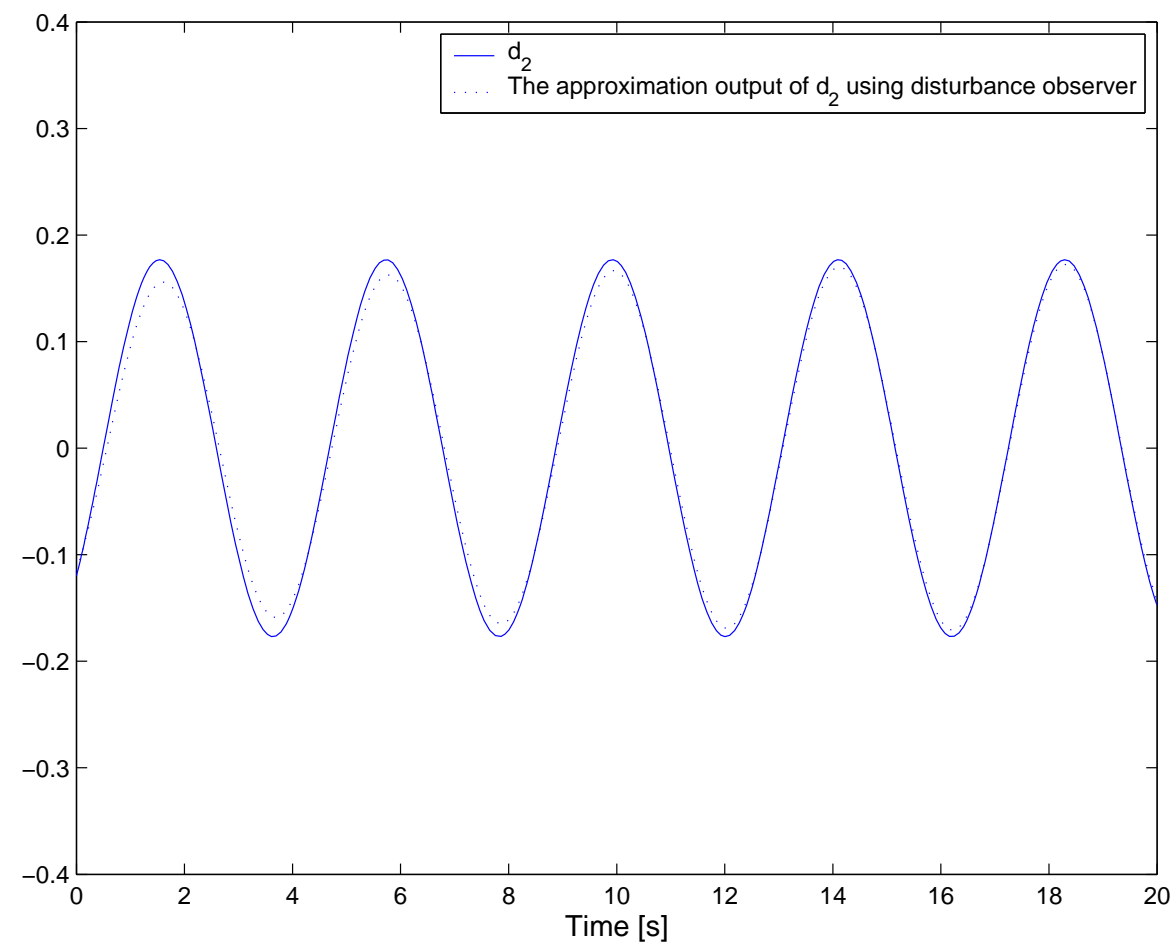

Figure 3: The disturbance $d_{2}$ and the approximation output of $\hat{d}_{2}$ 
scheme is valid.

\section{Acknowledgment}

This work is partially supported by Jiangsu Natural Science function (Granted Number: SBK2008390) and Aeronautical Science Foundation of China (Granted Number: 20075152014). The authors also gratefully acknowledge the helpful comments and suggestions of the reviewers, which have improved the presentation.

\section{References}

[1] J H Kim. Guaranteed cost control of parameter uncertain systems with time delay. International Journal of Control, Automation, and Systems. 2000, 2(1): 19 - 23

[2] J H Kim. Delay and its time-derivative dependent robust stability of time-delayed linear systems with uncertainty. IEEE Transaction on Automatic Control. 2001, 46(5):789-792

[3] M Wu, Y He, J H She, G P Liu . Delay-dependent criteria for robust stability of time-varying delay systems . Automatica. 2004, 40 (6): 1435-1439

[4] Yong He, M Wu , J H She, G P Liu. Delay-dependent robust stability criteria for uncertain neutral systems with mixed delays. Systems 83 Control Letters. 2004, 51 (1): 57-65

[5] Y H Roh, J H Oh. Robust stabilization of uncertain input-delay systems by sliding mode control with delay compensation. Automatica. 1999, 35 (11): 1861-1865

[6] C Y Kao, A Rantzer. Stability analysis of systems with uncertain time-varying delays. Automatica. 2007, 43 (6): $959-970$

[7] Q L Han . A new delay-dependent absolute stability criterion for a class of nonlinear neutral systems. Automatica. 2008, 44 (1) $272-277$

[8] T P Zhang, S S Ge. Adaptive neural control of MIMO nonlinear state time-varying delay systems with unknown dead-zones and gain signs. Automatica. 2007, 43 (6): 1021 - 1033

[9] L Yu, J Chu. An LMI approach to guaranteed cost control of linear uncertain time-delay systems. Automatica. 1999, 35 (6) :1155-1159 
[10] C H Lien. Non-fragile guaranteed cost control for uncertain neutral dynamic systems with timevarying delays in state and control input. Chaos, Solitons and Fractals. 2007, 31 (4): 889-899

[11] C H Lien. Delay-dependent and delay-independent guaranteed cost control for uncertain neutral systems with time-varying delays via LMI approach. Chaos, Solitons and Fractals. 2007, 33 (3): $1017-1027$

[12] H Z Li, S I Niculescu1, L Dugard J M Dion. Robust guaranteed cost control of uncertain linear time-delay systems using dynamic output feedback. Mathematics and Computers in Simulation. 1998, 45 (3): 349-358

[13] G P Lu, L F Yeung. $H_{\infty}$-control problem for linear systems with multiple time-delays via dynamic output feedback . Mathematics and Computers in Simulation. 2002, 60 (3):335-345

[14] X M Zhang, Q L Han. Robust $H_{\infty}$ filtering for a class of uncertain linear systems with timevarying delay . Automatica. 2008, 44 (1): 157 - 166

[15] D Ye, G H Yang. Adaptive reliable $H_{\infty}$ control for linear time-delay systems via memory state feedback. IET Control Theory Appl.. 2007, 1 (3): 713-721

[16] V Suplin, E Fridman, U Shaked . $H_{\infty}$ control of linear uncertain time-delay systems-a projection approach. IEEE Transaction on Automatic Control. 2006, 51(4):680-685

[17] J H Kim, D C Oh. Robust and non-fragile $H_{\infty}$ control for descriptor systems with parameter uncertainties and time delay. International Journal of Control, Automation, and Systems. 2007, $5(1): 8-14$

[18] Y S Lee, Y S Moon, W H Kwon, P G Park. Delay-dependent robust $H_{\infty}$ control for uncertain systems with a state-delay. Automatica. 2004, 40 (1): 65-72

[19] J D Chen. Robust $H_{\infty}$ output dynamic observer-based control of uncertain time-delay systems. Chaos, Solitons and Fractals. 2007, 31(2): 391-403

[20] L Yu, J Chu, H Y Su. Robust memoryless $H_{\infty}$ controller design for linear time-delay systems with norm-bounded time-varying uncertainty. Automatica. 1996, 32(12): 1759-1762

[21] J Huang. On the minimal robust servo-regulator for nonlinear systems. Systems $\&$ Control Letters. 1995; 26(3):313-320 
[22] E Kim. A discrete-time fuzzy disturbance observer and its application to control. IEEE Transaction on Fuzzy Systems. 2003; 11(3):399-410

[23] E Kim. A fuzzy disturbance observer and its application to control. IEEE Transaction on Fuzzy Systems. 2002; 10(1):77-84

[24] W H Chen. Disturbance observer based control for nonlinear systems. IEEE Transaction on Mechatronics. 2004; 9(4):706-710

[25] W H Chen, D J Ballance, P J Gawthrop, J O'Reilly. Nonlinear PID predictive controller. IEE Proc.-Control Theory Appl.. 1999; 146(6):603-611

[26] W H Chen. Nonlinear disturbance observer-enhanced dynamical inversion control of missiles. Journal of Guidance, Control, and Dynamics. 2003; 26(1):161-166

[27] W H Chen, D J Ballance, P J Gawthrop, J O'Reilly. A nonlinear disturbance observer for robotic manipulators. IEEE Transaction on Industrial Eleectronics. 2000; 47(4); 932-938

[28] A Isidori, C I Byrnes. Output regulation of nonlinear systems. IEEE Transaction on Automatic Control. 1990; 35:131-140 